Roosevelt, Churchill und Stalin beschlossen in Jalta aus den bereits erwähnten sicherheitspolitischen Motiven, Deutschland unter der Aufsicht eines Alliierten Kontrollrates vollständig zu entnazifizieren, $\mathrm{zu}$ entwaffnen und $\mathrm{zu}$ entmilitarisieren. Vorgesehen wurde, Deutschland in vier Besatzungszonen aufzuteilen, da Frankreich - zur Stabilisierung Westeuropas nach Kriegsende - in den Kreis der Sieger aufgenommen werden sollte. Mit Sitz in der gemeinsam verwalteten und in vier Sektoren aufgeteilten Stadt Berlin war der Alliierte Kontrollrat künftig das oberste Regierungs-, Kontroll-, Koordinations- und Verwaltungsorgan für Deutschland. Zudem einigte man sich, dass Deutschland insgesamt 20 Milliarden Dollar an Reparationen zu zahlen hat, von denen die Sowjetunion aufgrund der durch den Krieg angerichteten Zerstörungen die Hälfte bekommen sollte. Insbesondere der amerikanische Präsident zeigte an der Deutschlandfrage kein allzu großes Interesse. Einerseits wollte er angesichts der noch laufenden Kriegshandlungen mit dem »Hitlerregime« diesen Problemkomplex für später aufheben, andererseits fokussierte Roosevelt mehr sein Projekt der künftigen Weltorganisation der Vereinigten Nationen. Seine Hoffnungen, nach Beendigung des Zweiten Weltkrieges die Zusammenarbeit mit Stalin fortsetzen zu können, resultierten vor allem aus den positiven Diskussions- und Verhandlungserfahrungen an diesem Punkt. Denn Roosevelts Einschätzung zufolge war auch dem sowjetischen Staatsführer die Gründung einer gemeinsamen Weltorganisation zur Sicherung des Friedens zwischen den Staaten von großer Wichtigkeit. Denn:

Stalin stimmte ihm zu: Solange die jetzigen Regierungschefs lebten, würde keiner von ihnen sein Land in aggressive Handlungen verwickeln. Aber das sei in zehn Jahren schon anders. »Eine neue Generation würde in[s] Dasein treten, die nicht die Schrecken des gegenwärtigen Krieges kennen werde.« Man sei also verpflichtet, eine entsprechende Organisation zu schaffen, wenn man für fünfzig Jahre Frieden haben wolle. »Die größte Gefahr wäre der Konflikt zwischen den drei hier vertretenen Großmächten $[. .$.$] «Solange ihre Einigkeit erhalten bleibe, drohe wenig Gefahr für den Weltfrieden. { }^{99}$

\title{
2.2 Zunehmende Eskalation zwischen den Supermächten
}

Die Gegensätze zwischen den Alliierten sollten sich zuallererst an gänzlich divergierenden Vorstellungen über die Zukunft Polens entzünden. Auf der Grundlage der Atlantik-Charta präsentierte die amerikanische Seite den Entwurf einer Erklärung, aus der hervorging, dass in Zukunft alle Völker das Recht haben,

ihre eigene Regierungsform zu wählen, unter der sie leben wollen, die Wiederherstellung der souveränen Rechte und der eigenen Regierung für die Völker, die von den Aggressor-Staaten dieser Rechte zwangsweise beraubt wurden. »Die Einrichtung von Ordnung in Europa und der Wiederaufbau des nationalen wirtschaftlichen Lebens muß durch Vorgänge erreicht werden, die alle befreiten Völker instand setzen, die letzten Spuren von Nazismus und Faschismus zu zerstören und demokratische Institutionen ihrer eigenen Wahl zu errichten. ${ }^{100}$

99 Dülffer, Jalta, 4. Februar, S. 18.

100 Ebd., S. 22. 
In den Augen der Amerikaner und der Engländer konnte in Bezug auf eine Umwandlung zu demokratischen Verhältnissen nur eine parlamentarische Demokratie westlicher Couleur gemeint sein. Für die totalitäre Sowjetunion bestand ein grundsätzlicher ideologischer Widerspruch zu den westlichen demokratisch-kapitalistischen Systemen. Gleichwohl hatte Stalin keinerlei Bedenken, die von der US-Regierung verfasste Erklärung zu unterschreiben - die das Prinzip der freien und ungehinderten Wahlen vorsah -, da die tatsächliche Umsetzung in seinen Augen nach sowjetischer Interpretation erfolgen würde und dies vor allem von den politischen Kräfteverhältnissen abhinge. Für Stalin bedeutete dies, so der Historiker Leonid Luks:

dass die UdSSR nur denjenigen politischen Kräften Osteuropas die Teilnahme an politischen Entscheidungsprozessen gestattete, die aus ihrer Sicht »demokratisch « und nicht »volksfeindlich« waren. Und in die Kategorie der »volksfeindlichen Kräfte« sollte im Laufe der Zeit eine immer größere Zahl von politischen Gruppierungen geraten, bis schließlich nur die Kommunisten und ihre Marionetten auf der politischen Bühne übrigblieben. Gegen dieses Vorgehen wurde seitens der Westmächte unentwegt protestiert. $^{101}$

Sicherlich war sich insbesondere Roosevelt zuwenig der kontradiktorischen westlichen sowie östlichen Gesellschaftssysteme gewahr, dachte und handelte zu wenig im Kontext der machtpolitischen Situation. Ansonsten hätte er zweifelsohne erkennen müssen, dass es niemals zur wirklichen Umsetzung seiner Erklärung kommen würde. Denn die Sowjetunion wollte zum einen niemals freiwillig auf einmal militärisch eroberte Gebiete verzichten. Zum anderen wollte sich Stalin immerfort das absolute politische Entscheidungsmonopol und die totale Kontrolle über diese Länder vorbehalten. Der der Ambivalenz der Anti-Hitler-Koalition entspringende Konflikt kristallisierte sich zuerst an dem »Problem« Polen heraus, der erst nach Beendigung der Konferenz in Jalta völlig sichtbar wurde.

Die Sowjetunion setzte infolge der territorialen Gewinne, die ihr im Rahmen des Rückzuges der deutschen Truppen zufielen, beim Überschreiten der Vorkriegsgrenze eine kommunistische polnische Regierung ein. Die gegen den Willen eines großen Teiles der polnischen Bevölkerung an die politische Schaltzentrale gebrachte Regierung bestand aus Moskau untergebenen prokommunistischen und kommunistischen Personen: dem sogenannten Lubliner Komitee. Auf der anderen Seite erkannten die Vereinigten Staaten und England die polnische Exilregierung in London an. Die polnische Exilregierung kündigte an, das sogenannte Katynverbrechen zu untersuchen, das dafür sorgte, dass die polnisch-sowjetischen Beziehungen in eine schwere Krise stürzten. Am 13. April 1943 wurden nämlich in dem polnischen Dorf in der Nähe von Smolensk von deutscher Seite Massengräber von schätzungsweise 12000 polnischen Offizieren gefunden. Die nationalsozialistische Propaganda beschuldigte die Sowjetunion, die tatsächlich im Frühjahr 1940 - also vor dem deutschen Überfall auf die Sowjetunion - in russische Kriegsgefangenschaft geratenen polnischen Offiziere ermordet zu haben. Die Sowjetunion bestritt die Verantwortung für das Massaker und behauptete, es wäre ein deutscher Versuch, die Anti-Hitler-Koalition zu spalten. Ein neutrales Untersuchungsergebnis erbrachte, dass das Massaker von sowjetischen

101 Luks, Geschichte Russlands und der Sowjetunion, S. 395. 
Stellen - von NKWD-Einheiten - begangen wurde. Daraufhin brach die sowjetische Regierung die Beziehungen zur polnischen Exilregierung ab. ${ }^{102}$

Stalin verhandelte in Jalta, in Anbetracht der Tatsache, dass seine Truppen den überwiegenden Teil Polens bereits erobert hatten, aus einer Position der Stärke. Er gab seinen Verhandlungspartnern Roosevelt und Churchill - aus dessen Wahrnehmung heraus der sowjetische Vormarsch in Osteuropa zu neuen kommunistischen Diktaturen führen wird und mit weiteren Expansionen Stalins in Richtung auf das restliche Europa zu rechnen sein dürfte - unmissverständlich zu verstehen, dass er die durch den deutsch-sowjetischen Nichtangriffspakt von 1939 resultierenden Gebietsgewinne, d.h. Ostpolen, als seine politische Beute betrachtete, sodass die Alliierten eine polnische Westverschiebung nach Westen vorsahen, die - wie es bereits in Teheran entschieden wurde - auf Kosten Deutschlands gehen sollte. Als polnische Ostgrenze sahen die Alliierten, der sowjetischen Forderung entsprechend und gegen den Willen der polnischen Exilregierung, die sogenannte Curzon-Linie vor. »Die Oder-Grenze war akzeptiert. Die Sowjetunion insistierte aber auf der Oder-Neiße-Linie und meinte damit die westliche Neiße. ${ }^{103}$

Eine abschließende Regelung sollte, was diesen Punkt anbelangt, auf der späteren Friedenskonferenz erfolgen, die nach dem Ende des Krieges in Potsdam stattfand. So sehr unter anderem die alliierte Polenpolitik auch weiterhin politischen Zündstoff enthielt und unter den Teilnehmern für Spannungen sorgte - beispielsweise durch die infolge der polnischen Westverschiebung millionenfach vertriebenen Deutschen in die westliche Zone -, so sehr wurde das sich zusehends zugunsten der Sowjetunion entwickelnde Problem überlagert durch den Versuch, eine grundsätzliche Nachkriegsordnung zu errichten. Anders gesagt: Die Deutschlandfrage dominierte auf der nach Kriegsende anberaumten Potsdam-Konferenz gegenüber den Fragen der konkreten Gestaltung der Staaten, die sich im Einzugsbereich der Sowjetunion befanden. Gleichwohl waren dieser Problemkomplex und die Deutschlandfrage ständig ineinander verschachtelt. Seit 1945/46 führten die zunehmenden Brüche der auf der Jalta-Konferenz schriftlich vereinbarten Abmachungen über die ost- und südeuropäischen Länder durch den sowjetischen Staatsführer dazu, dass der Kalte Krieg sich zuspitzte und auf die Deutschlandfrage ausstrahlte. Die sich allmählich Bahn brechende Sowjetisierung zeigte sich - wie bereits angesprochen wurde - an der bewussten Missachtung Moskaus, die Etablierung demokratischer Systeme mit freien Wahlen im Staatengürtel von Polen bis Bulgarien zu akzeptieren. Tatsächlich verwandelte Stalin diesen Staatengürtel unter anderem mit extremen Repressionsmethoden zu einem Bestandteil seines sowjetischen Macht- und Interessensystems.

Trotz der grundsätzlichen gesellschaftlichen und politischen Systemgegensätze kann zweifelsohne in Hinblick auf die tatsächliche Nachkriegspolitik der Vereinigten Staaten und der Sowjetunion - die nach dem Sieg gegen Deutschland die einzig verbliebenen Supermächte waren und die als die eigentlichen Exponenten ihres jeweiligen Lagers im Ost-West-Gegensatz agierten - von einer offenen geschichtlichen Situation gesprochen werden. Das heißt, wenn auch der Kalte Krieg dem politischgesellschaftlichen Systemgegensatz der Bündnispartner quasi immanent war und der Konflikt im Laufe der Zusammenarbeit in der Anti-Hitler-Koalition immer schon

102 Siehe Dülffer, Jalta, 4. Februar 1945, S. 147 f.

103 Ebd., S. 26. 
latent vorhanden war, eskalierte der Konflikt zwischen den beiden Supermächten nicht gleich nach Kriegsende, sondern Schritt für Schritt erst ab etwa 1947/48. In der Wahrnehmung des westlichen Lagers spielte - neben der erwähnten Etablierung des sowjetischen Herrschaftssystems in Ost- und Südosteuropa; die Länder wurden später als Satellitenstaaten apostrophiert - die als unmittelbare Bedrohung empfundene kommunistische Expansionspolitik in Richtung auf das übrige Europa die entscheidende Rolle. Im Zentrum des Ost-West-Konfliktes stand aus geopolitischen Gründen Deutschland. Hier prallten die weltanschaulichen Konzepte der beiden Hauptkontrahenten direkt aufeinander. Im Mittelpunkt der Rivalität zwischen den Vereinigten Staaten und der Sowjetunion stand Berlin. Rückblickend schreibt der deutsche Historiker Josef Foschepoth:

Im Zuge der raschen Verschlechterung der Beziehungen zwischen den Mächten nach dem Krieg wurde Deutschland zum eigentlichen Schauplatz jener Auseinandersetzungen, die gemeinhin als Kalter Krieg bezeichnet werden. Aus dem Kampf gegen Deutschland war ein Kampf um Deutschland geworden. Zwar war die Deutsche Frage nicht die Ursache des Kalten Krieges, doch besteht zwischen beiden ein direkter Zusammenhang. ${ }^{104}$

\subsection{Unterschiedliche Vorstellungen der Besatzungsmächte bei der konkreten Deutschlandpolitik}

Die bedingungslose Kapitulation vom 8. Mai 1945 bedeutete nicht nur das Ende des Naziregimes, sondern hatte auch konsequenterweise den Verlust deutscher Staatlichkeit und mithin deutscher Souveränität zur Folge. Dieses staatsrechtliche Vakuum wurde am 5. Juni 1945 beendet, als die vier Besatzungsmächte - vertreten durch die vier jeweils für ihre Zone zuständigen Oberbefehlshaber General Eisenhower (USA), Marschall Schukow (UdSSR), Feldmarschall Montgomery (Großbritannien) sowie General de Lattre de Tassigny (Frankreich) - in der "Erklärung in Anbetracht der Niederlage Deutschlands« öffentlich bekannt gaben, dass von dem Tage an die oberste Regierungsgewalt in dem Land von den Vertretern der vier alliierten Siegerstaaten übernommen und von ihnen gemeinsam ausgeübt werde. ${ }^{105}$

Tatsächlich sollten jedoch die wichtigsten Fragen in Bezug auf die alliierte Nachkriegspolitik im besetzten Deutschland auf der vom 17. Juli bis zum 2. August 1945 dauernden Potsdamer Konferenz thematisiert und geklärt werden. Die Regierungschefs der drei Siegermächte - die Vereinigten Staaten wurden von dem erstmals auf der internationalen Bühne hervortretenden neuen Präsidenten Harry Truman vertreten - erzielten in einigen Grundsatzfragen eine Übereinkunft. Das in die vier Besatzungszonen aufgeteilte Deutschland - einschließlich Berlins - sollte als eine wirtschaftliche Einheit behandelt werden. »Da außerdem«, so der deutsche Historiker Hermann Graml, »die baldige Errichtung deutscher Zentralbehörden - als Hilfsorgane des Alliierten Kontrollrates - vereinbart wurde, sah es in der Tat so aus, als sei

104 Josef Foschepoth (Hg.), Kalter Krieg und Deutsche Frage. Deutschland im Widerstreit der Mächte 1945-1952, Göttingen/Zürich 1985, Einleitung S. 11-31, hier S. 11, vgl. grundsätzlich hierzu Ernst Nolte, Deutschland und der Kalte Krieg, Stuttgart 1985. 